\title{
Die chilensischen Aphodiden
}

von

E. v. Harold in München.

Der eigenthümliche Charakter der chilensischen Fauna, der sich fast in allen Familien der Coleopteren ausspricht, am auffallendsten vielleicht bei den Carabiden, Lucaniden, Melolonthiden und Heteromeren, läfst sich auch in den sonst unscheinbaren Formen der Aphodiden nicht verkennen, und eine Zusammenstellung des bisher in dieser Gruppe bekannt Gewordenen dürfte nicht-ohne einiges Interesse sein.

An ächten Aphodien scheint übrigens Chili, wie Süd-Amerika überhaupt, arm zu sein; selbst von den wenigen Arten, welche der Gattung Aphodius beizuzählen sind, zeigt fast jede etwas Fremdartiges, so dafs typische Aphodien, wie sie in Nord-Amerika und selbst noch in Mexiko sich finden, hier ganz zu fehlen scheinen.

Da die chilensischen Aphodiden zum Theil auch in die Unterabtheilungen der Psammodiden und Ataeniden gehörên, so gebe ich hier, einer speciellen Bearbeitung der Aphodiden, welche ich für meine coleopterologischen Hefte beabsichtige, vorgreifend, eine Uebersicht der Gruppen, in die sich die Gesammtmenge der Aphodiden theilen läfst.

1. Tibiae posticae calcaribus apicalibus brevissimis . Pseudaphodidae.

Tibiae posticae calcaribus longitudine normali 2

2. Mandibulae patentes . . . . . . . . . . 3

Mandibulae occultae. . . . . . . . . . 4

3. Pygidium obtectum . . . . . . Aegialidae.

Pygidium liberum . . . . . . Chironidae.

4. Tibiae posticae transversim carinatae. . Aphodidae.

Tibiae posticae simplices, absque carinis . 5

5. Caput asperato-granosum . . . . . Psammodidae.

Caput laeve vel tantum rugose-punctatum Ataenidae. 
Nur aus den lelzteren drei Gruppen finden sich Repräsentanten in Chili; die Totalsumme der dort aufgefundenen Arten beträgt bis jelzt 13 Arten, von welchen je 5 auf die Gattungen Aphodius und Alcenius kommen, 2 auf Psammodius, 1 auf Pleurophorus. Selbst von dieser geringen Anzahl sind wieder 4 Arten in sofern in Abzug zu bringen, als die eine, Aphodius chilensis Sol., höchstwahrscheinlich identisch mit Ataenius gracilis Mels. sein dürfte, und die anderen drei, $\boldsymbol{P l}$. caesus, Aph. granarius und der zuletzt genannte Ataenius wohl nur als hospitirende Fremdlinge zu betrachten sind.

Ich gebe hier das Verzeichnifs dieser Arten, unter welchen sich zugleich noch einige neu zu beschreibende befinden:

\section{Aphodidae.}

\section{Aphodius granarius $\mathrm{L}$.}

Scarab. granarius L. Syst. Nat. I. 2. p. 547.

Ich erhielt von Herrn Fairmaire Stücke aus Chili dieser über den ganzen Erdkreis verbreiteten und vielfach benannten Art.

2. A. rugosiceps Harold.

A. rugosiceps Har. Berl. Ent. Ztschr. 1859 p. 213.

A. chilensis Erichs. IIus. Berol.

Diese Art weicht durch ziemlich stark gewölbten Unterleib, deutlich vierzähnige Vorderschienen, etwas rauhgekörneltes Kopfschild und bedeckte Augen nicht unerheblich von den typischen Gattungsgenossen $a b$, von denen sie aber doch wegen der deutlichen Querleisten der Hinterbeine nicht getrennt werden kann. Die Art kommt vielleicht auch in Peru vor, wenigstens tragen einige Stücke aus der Reich'schen Sammlung diese Heimathsangabe.

3. A. fulviventris Fairm.

A. fulviventris Fairm. Rev. zool. 1860 p. 268.

Eine durch flache Gestalt, kurzes und gerundetes Halsschild, die leicht nach rückwärts gerichteten Wangenzipfel und den Geschlechtsunterschied höchst ausgezeichnete Art. Bei dem Weibchen zeigen nämlich die Vorderschienen die gewöhnlichen drei Randzähne, die etwas schmäleren Schienen des Männchens sind aber nur zw e i zähnig.

\section{A. gracilipes nov. sp.}

Simillimus $A$ peruano Er., at minor, elytris profundius striatis thoraceque densius ac fortius punctato facile dignoscendus. Elongatulus, depressus, subnitidus, setiger, niger, elytris luteo-testaBerl. Entomol, Zeitschr. XI. 
ceis longitudinaliter fusco-subtesselatis. Caput irregulariter punctatum, genis sat prominulis. Thorax dense punctalus, punctis magnitudine valde inaequalibus, basi submarginatus, longitudinaliter obsolete impressus, lateribus rotundatis. Elytra striatu, striis crenalopunctatis, interstitiis 3,5 et 7 leviter convexis, seriatim obsoletissime subtuberculatis, reliquis panis, serie punctorum piliferorum. Corpus subtus obscure piceo-rufum, pedibus rufescentibus, gracilibus, tarsis posticis elongalis. - Long. $3 \frac{4}{5}$ lin.

Ein einzelnes Weibchen im Berliner Museum. Die Art vermittelt so ziemlich den $\boldsymbol{A}$. peruanus mit $\operatorname{dem} \boldsymbol{A}$. fulviventris; von ersterem unterscheidet sie die dichte und tiefe Punktirung des Halsschildes, von letzlerem die Färbung und die eigenthümliche Sculptur der Flügeldecken.

5. A. flaveolus nov. spec.

Elongatulus, leviter convexus, nitidulus, rufo-leslaceus, thorace elytrisque flavo-testaceis, sulura thoracisque disco plerumque infuscatis. Caput muticum, genis sat acutis, antice a clypeo vix distinctis, clypeo medio truncato, late subemarginato. Thorax marginibus sat longe cilialus, punctatus, basi immarginatus, angulis posticis rotundatis. Elytra sat profunde striala, striis parum distincte punctatis, interstitiis laevibus, leviter convexis. Corpus subtus pallido-testaceum, antennis, tibiis et tarsis rufescentibus; tibiis posticis setis apicalibus inaequaliter longis, obsolete tantum carinatis. - Long. $1 \frac{3}{4}-2$ lin.

Von Herrn Dr. Kraatz freundlichst mitgetheilt. Eine kleine, dem $\boldsymbol{A}$. lividus 01. ähnliche, aber viel flachere Art, die noch ganz die Kopfbildung der ächten Aphodien hat, im Fufsbau aber schon die Neigung verräth, den Ataenien sich anzuschliefsen, da die Querleisten fast nur mehr an den mittlern Schienen zu erkennen sind.

\section{Ataenidae.}

6. A. Derbesis Sol.

Aphodius Derbesis Sol. Gay Hist. Chil. V. p. 72. t. 16. f. 1. (1851).

Wieder eine sehr eigenthümliche Form, die auch in der Gattung Ataenius, wohin sie die einfachen hinteren Schienen bringen, ziemlich isolirt steht. Die Art ist an den ziemlich langen Borsten, die den Seitenrand und zum Theil auch noch die Basis des Halsschildes bekleiden, dem runzlig - punktirten Kopfschilde und dem schwach gekrümmten ersten Tarsengliede der Hinterbeine leicht zu erkennen. 
7. A. stercorator $\mathrm{F}$ abr.

Aphodius stercorator Fabr. Syst. El. I. p. 81.

Oxyomus bonariensis Dej. Cat. 3 ed. p. 162.

Ein durch fast ganz Amerika verbreiteter Käfer, der unter der grofsen Menge sehr nah verwandter Gatlungsgenossen doch immer an den seitlich und hinten punktirten Flügeldecken gut zu erkennen ist.

8. A. picinus nov. sp.

Simillimus $A$. stercoratori, at elytris lateribus apiceque non punctatis, metatarsoque breviore dignoscendus. Nigro-piceus, nitidus, elongatus, subcylindricus. Thorax punctis majoribus ad latera et postice parum dense adspersus, angulis posticis rotundalis. Elytra profunde crenato-striata, interstitiis convexis, laevibus. Pedes rufo-picei, tarsorum posticorum arliculus primus calcari superiore nonnihil brevior.

Von Herrn Dr. Kraatz. A. stercorator, welcher dieser Art höchst ähnlich ist, unterscheidet sich durch dicht und ziemlich gleichmäfsig punktirtes Halsschild, etwas minder gewölbte Gestalt und die am Aufsenrande sehr deutlich punktirten Zwischenräume.

9. A. gracilis Mels.

Rhyssemus gracilis Mels. Proc. Ac. Phil. II. p. 137.

Psammodius gracilis Jacq. Duv. Hist. Cub. VII. p. 119.

Dieser schlanke und schmale, im Habitus dem Pleurophorus caesus nicht unähnliche Ataenius ist von Pennsylvanien über die westindischen Inseln bis nach Columbien, Peru nnd Chili verbreitet. Höchstwahrscheinlich ist mit ihm zu vereinigen der mir unbekannte

10. A. chilensis Sol.

Aphodius chilensis Sol. Gay Hist. Chil. V. p. 72.

\section{Psammodidae.}

11. Pleurophorus caesus $\mathrm{P}$ anz.

Scarab. caesus Panz. Faun. Germ. 35. 2.

Aphod. angustus Philippi Stett. Ztg. 1864 p. 316.

Dieser Käfer kommt ebenfalls im ganzen nördlichen Afrika, im angrenzenden Asien, in Nordamerika und in Chili vor; aus Philippi's Beschreibung geht schon die Identität seines $\boldsymbol{A}$. angustus mit $\boldsymbol{P}$. caesus sattsam hervor; auf gestellte Anfrage bestätigte mir freundlichst Herr C. A. Dohrn, welcher im Besitze der Typen sich befindet, meine desfallsige Vermuthung. 
12. Psammodius nanus De Geer.

Scarab. nanus De Geer Mem. Ins. IV. p. 318.

Psamm. parvulus Chevr. Ann. Fr. 1864 p. 415.

Ebenfalls weit verbreitet. Es liegen mir Stücke aus Mexiko, Cuba, New-York und Valparaiso vor. Dieses zierliche, walzenförmige Käferchen ist wahrscheinlich die kleinste Art unter allen Lamellicornien.

13. P. cruentus nov. spec.

Convexus, nitidulus, subelongatus, postice parum dilatatus, rufus, capite thoraceque nonnihil infuscatis. Caput asperato-granulatum, clypeo bidentato. Thorax setis marginalibus simplicibus, non clavatis, irregulariter subfoveolatim punctatus, absque sulcis transversis. Elytra sat profunde striata, margine laterali ciliata. Tibiae posticae apicem versus valde dilatatae, calcaribus apicalibus latis, tarsorum posticorum articulo primo apicem versus dilatalo. Long. 2 lin.

Von Herrn Dr. Kraatz mitgetheilt. Durch den Fufsbau ein ächter Psammodius, von den Gattungsverwandten leicht durch den vollständigen Mangel der Querwulsten auf dem Halsschilde zu unterscheiden; selbst Psam. laevicollis Klug, bei dem dieselben am schwächsten ausgeprägt sind, zeigt nach hinten eine sehr deutliche Längsvertiefung und beiderseits eine abgekürzte Querfurche.

Die Anzahl der chilensischen Aphodiden dürfte sich immerhin noch vermehren, wenn nur einmal diesen unansehnlichen Thieren mehr Aufmerksamkeit zugewendet wird; ich hätte schon bei dieser Gelegenheit noch 2 weitere Ataenius-Arten beifügen können, von welchen die eine dem $\boldsymbol{A}$. Derbesis nahe steht; es ist mir aber weder ihre Herkunft sicher verbürgt, noch ladet der schlechte $\mathrm{Zu}$ stand, in dem sie sich befinden, zur Beschreibung ein. 


\section{$2 \mathrm{BHL}$ Biodiversity Heritage Library}

Harold, Edgar von. 1867. "Die chilensischen Aphodiden." Berliner entomologische Zeitschrift / herausgegeben von dem Entomologischen Vereine in Berlin 11(304)278-282. https://doi.org/10.1002/mmnd.18670110307.

View This Item Online: https://www.biodiversitylibrary.org/item/36408

DOI: https://doi.org/10.1002/mmnd.18670110307

Permalink: https://www.biodiversitylibrary.org/partpdf/210209

\section{Holding Institution}

Smithsonian Libraries

\section{Sponsored by}

Smithsonian

\section{Copyright \& Reuse}

Copyright Status: Public domain. The BHL considers that this work is no longer under copyright protection.

This document was created from content at the Biodiversity Heritage Library, the world's largest open access digital library for biodiversity literature and archives. Visit BHL at https://www.biodiversitylibrary.org. 